\title{
A Rare Case of Complete Uterine Torsion in a Postmenopausal Woman
}

\author{
Ping Ling $\mathrm{Ho}^{\mathrm{a}, \mathrm{c}}$, Mohamed Siraj Bin Shahul Hameed ${ }^{\mathrm{b}}$, \\ Bernard Chern ${ }^{\mathrm{b}}$
}

\begin{abstract}
We present a rare case of uterine torsion caused by an 18 -cm subserosal myoma weighing $3.9 \mathrm{~kg}$ in a postmenopausal lady in a tertiary center. A 52-year-old postmenopausal lady presented with abdominal pain and no bowel movements of 3 days duration. Multiplanar computed tomography (CT) of abdomen and pelvis showed uterine torsion, while initial pelvis ultrasound was unremarkable. Patient underwent laparotomy and was found to have torsion of the uterus at the level of the isthmus with a large subserosal myoma. She underwent total hysterectomy and bilateral salpingo-oophorectomy. Post-operatively, patient recovered well. Subserosal myoma causing uterine torsion is extremely uncommon, but in a known case of large subserosal myoma in a postmenopausal lady, high index of suspicion is needed when they present with acute abdominal pain. However, prompt identification with advanced radiological imaging is important to minimize associated morbidity and mortality.
\end{abstract}

Keywords: Gynecological emergency; Multiplanar computed tomography; Non-gravid uterus; Uterine torsion

\section{Introduction}

Uterine torsion is a rare gynecological emergency. It commonly occurs in women of reproductive age group, and more commonly in a gravid uterus. It is part of spectrum of surgical emergency, potentially life-threatening and may result in rapid clinical deterioration, due to underlying irreversible ischemia and potentially gangrenous changes to the uterus. A prompt and accurate diagnosis is therefore important to reduce the mortality and morbidity. We present a rare case of uterine torsion caused by a myoma weighing $3.9 \mathrm{~kg}$ in a postmenopausal lady, emphasizing the importance of high clinical suspicion

Manuscript submitted May 17, 2019, accepted May 31, 2019

aDepartment of Obstetrics and Gynaecology, KK Women's and Children's Hospital, Singapore

bDepartment of Minimally Invasive Surgery, KK Women's and Children's Hospital, Singapore

${ }^{\mathrm{c} C o r r e s p o n d i n g ~ A u t h o r: ~ P i n g ~ L i n g ~ H o, ~} 13$ Bartley Road, \#02-21 The Quinn, 539789 Singapore, Singapore. Email: pingling.ho@gmail.com

doi: https://doi.org/10.14740/jmc3314 and advanced radiological imaging in its management.

\section{Case Report}

A 52-year-old postmenopausal, single lady presented with abdominal pain and no bowel movements of 3 days duration. She had no associated nausea and vomiting. She reported a similar episode 5 months prior to presentation which lasted for 4 days and resolved spontaneously. Initial examination revealed distended abdomen with a firm mass measuring 40 weeks uterine size, tender on palpation. Abdomen otherwise was soft and non-tender. No rebound tenderness or guarding was found. She was stable on presentation. She was afebrile, heart rate was $71 \mathrm{bpm}$ and blood pressure was 181/80 $\mathrm{mmHg}$. Hemoglobin and white cell count on admission were normal at $15.7 \mathrm{~g} / \mathrm{dL}$ and $7,200 / \mathrm{mm}^{3}$, respectively. Tumor markers level of beta human chorionic gonadotropin (beta-hCG), carcinoembryonic antigen (CEA), CA125 and alpha-fetoprotein (AFP) were all within normal range and chest X-ray was reported normal. Computed tomography (CT) of abdomen and pelvis with multiplanar and coronal reformation showed torsion of the uterus and bilateral adnexa (Figs. 1 and 2), while initial pelvis ultrasound was unremarkable. Bilateral ureters were assessed to be not involved. Patient underwent laparotomy, intraoperatively noted normal sized uterus torted $720^{\circ}$ at the level of the isthmus containing multiple uterine fibroids with the largest measuring $18.5 \mathrm{~cm}$ and bilateral adnexa torsion (Figs. 3 and 4). The tissues appeared ischemic after detorsion (Fig. 5). Considering the age, menopausal status and size of the fibroid and ischemic uterus, she underwent total hysterectomy and bilateral salpingo-oophorectomy. Histopathology described uterine size $60 \times 40 \times 160 \mathrm{~mm}$, containing multiple myoma, largest $180 \mathrm{~mm}$, weighing $3,927 \mathrm{~g}$. Bilateral ovaries measure $30 \times 20 \mathrm{~mm}$. Left seromucinous cystadenoma and focal infarction of uterus were reported. No evidence of malignancy. Postoperatively, patient recovered well.

\section{Discussion}

Uterine torsion is defined as rotation of at least $45^{\circ}$ on its long axis, commonly dextrorotation. Uterine torsion is rare, and incidence and prevalence of uterine torsion is not well known. Majority of cases of uterine torsion reported are associated with pregnancy, often asymptomatic and only found during 

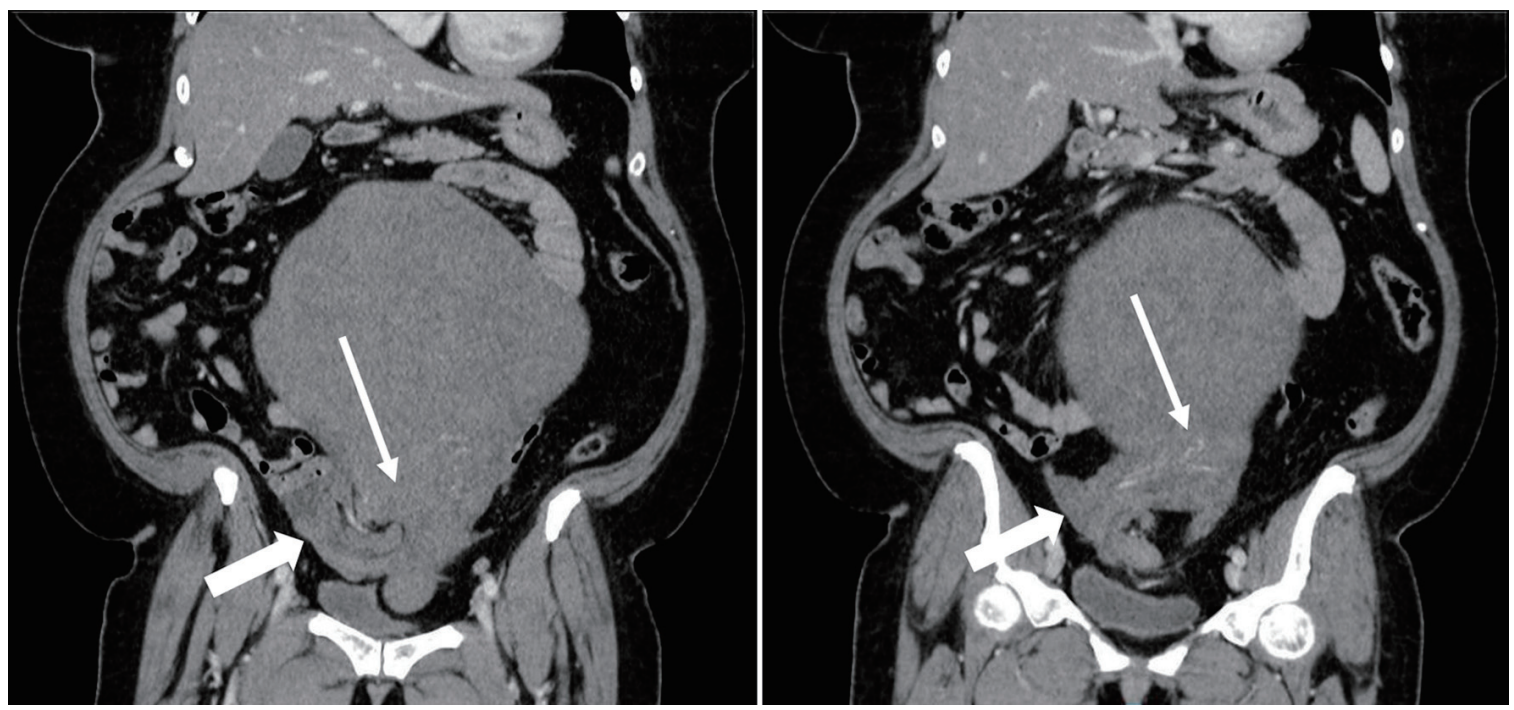

Figure 1. Contrast-enhanced CT of abdomen and pelvis (coronal section) showed torsion of lower uterine segment (white arrow) and bilateral adnexa at the right hemipelvis (blocked white arrow).

cesarean section. This condition can occur in all ages, all parity and in any trimester of the pregnancy. It is commonly associated with fetal malpresentation, others including uterine anomalies such as bicornuate uterus. Detorsion of uterus is usually attempted prior delivery, however, if it is not possible, delivery can be safely made via a longitudinal incision at the posterior wall of the uterus. Maternal recovery does not differ from routine cesarean section, however, it may experience higher perinatal mortality in future pregnancies.

Torsion of a non-gravid uterus is even rarer. A literature search reveals less than 50 reported cases of uterine torsion in a non-gravid uterus. Torsion of a non-gravid uterus has been reported most commonly associated with large uterine fibroid, others in association with congenital uterine anomalies, such as bicornuate uteri and large ovarian tumor, more often than not, associated with an adnexa torsion. Rarely, pelvic adhesion has also been found in association. The underlying pathophysiology of the non-gravid uterine torsion is not well known. We postulate that laxity and elongation of the supporting tissues and ligaments caused by the large subserosal fibroids may increase the risk of uterine torsion against the well anchored cervix to the lateral wall by the cardinal and uterosacral ligaments compared to lateral attachment of the uterus. The asymmetrical enlargement of uterus by the myoma or by the unilateral large adnexa mass may also result in deviation of axis and subsequent rotation of the uterus along its longitudinal axis. Most common point of torsion of the uterus was reported to have occurred at the uterine isthmus level.

There is no specific or pathognomonic symptom as of date to diagnose uterine torsion $[1,2]$. Symptoms of varying degree, onset and severity have been reported in uterine torsion. Abdominal pain is reported to be the most common presenting symptom. Pain may vary from mild to acute and severe, with or without shock symptoms. Other symptoms of genitourinary or gastroistestinal symptoms as well as per vaginal bleeding have also been reported. Some remained asymptomatic [3, 4].

Most cases reported in the literature were also diagnosed intra-operatively. To the best of our knowledge, our case is the
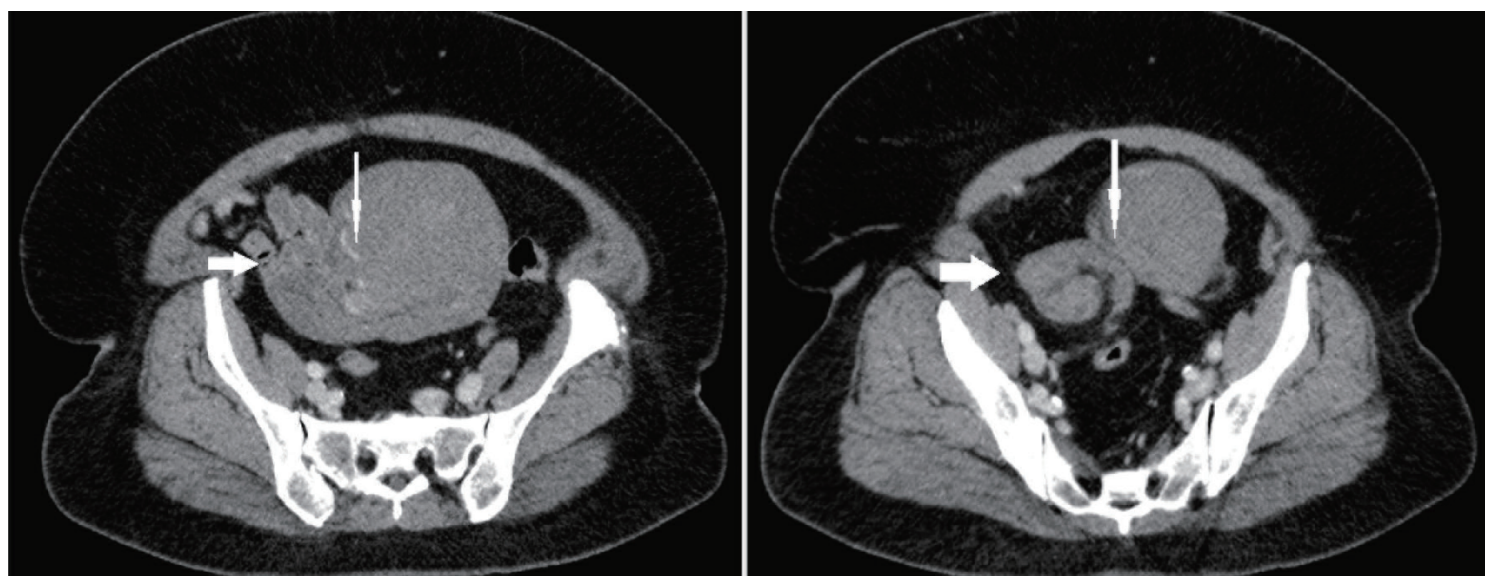

Figure 2. Sagittal section of contrasted multiplanar CT of abdomen and pelvis. 


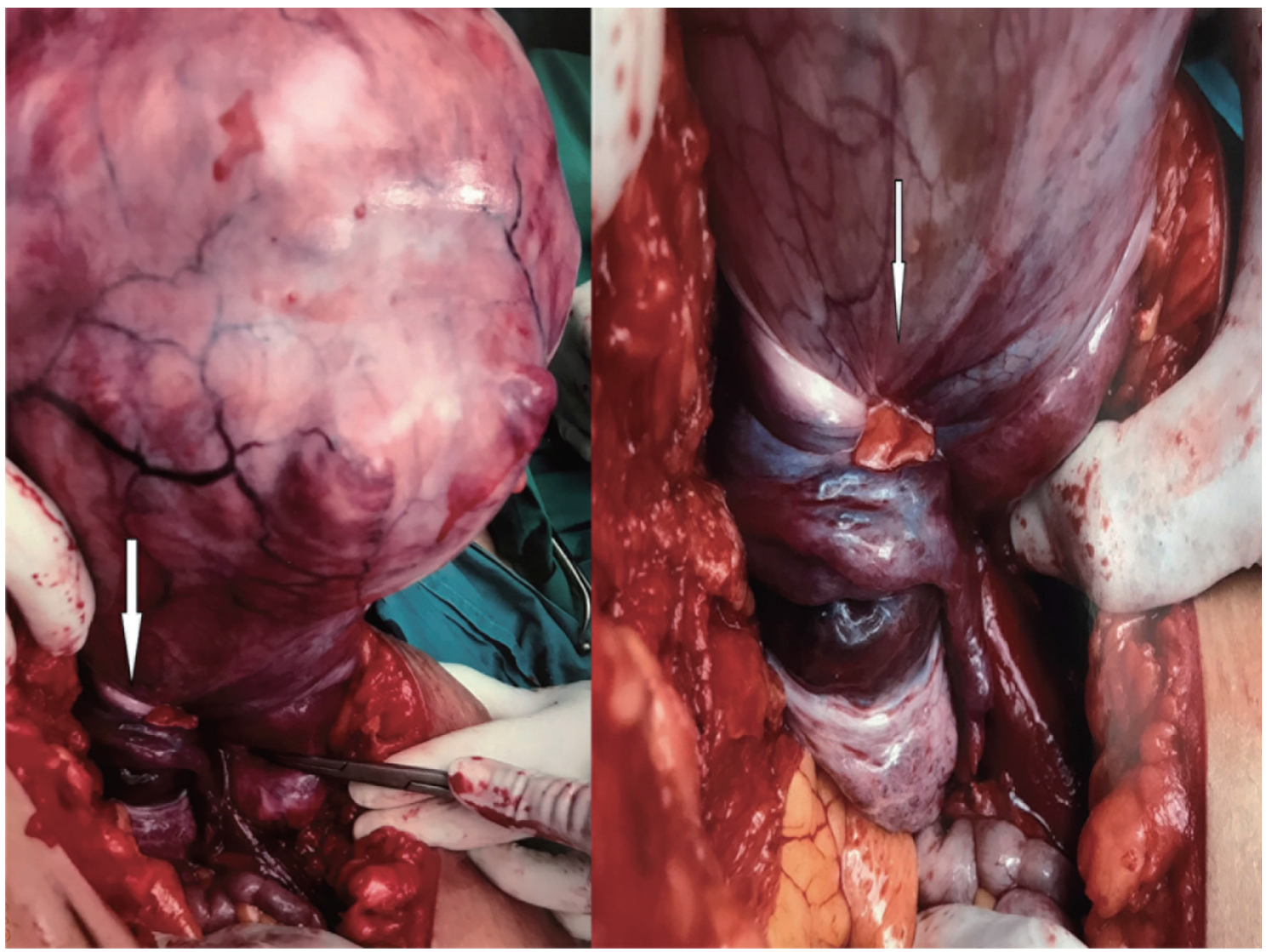

Figure 3. Uterus with large subserosal fibroids torted at the level of the isthmus, which involved bilateral adnexa (as indicated with arrow).

first case with a pre-operative diagnosis to be reported, and the first to describe the features of uterine torsion on CT of the abdomen and pelvis. In view of the non-specific clinical signs and symptoms, imaging plays an utmost important role to aid diagnosis making in this situation. Multiplanar images in standard sagittal and coronal planes are useful in the evaluation of both

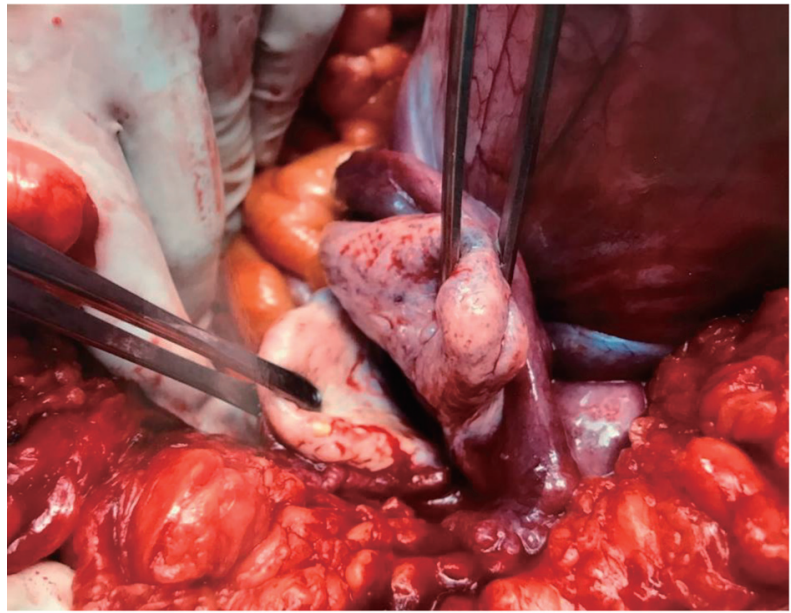

Figure 4. Right side adnexa torted towards left side and both adnexa seen at the same side (left side of pelvis).

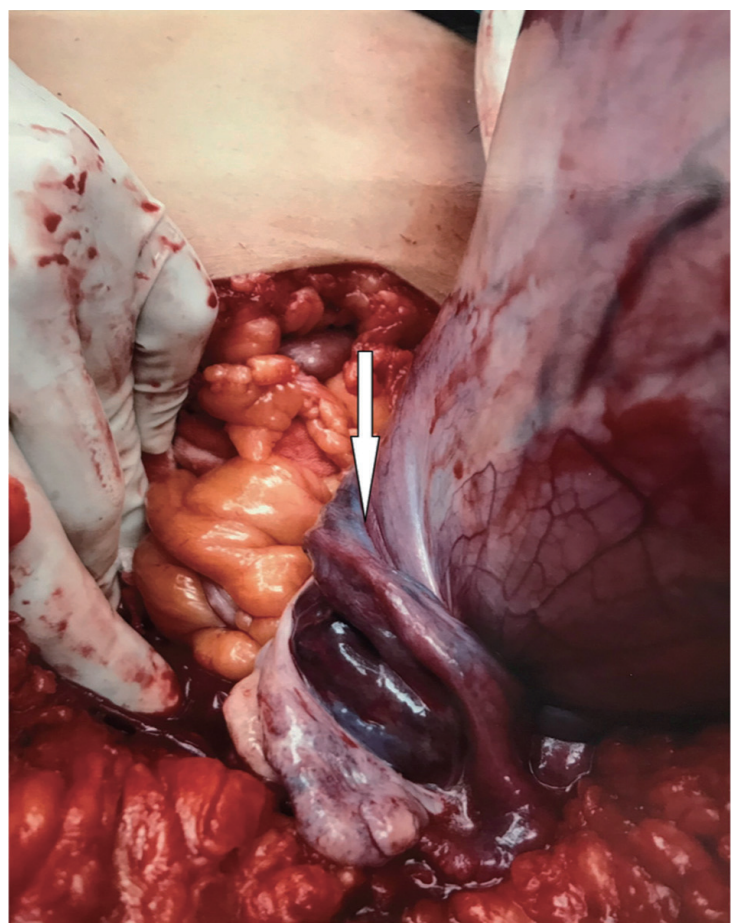

Figure 5. Congested torted adnexa after detorsion. 
normal and variant female pelvis anatomy. A whorled structure is pathognomonic for adnexal torsion. Gas in uterine cavity has also been described, likely secondary underlying necrosis [5, $6]$. X-sign configuration of the upper vagina was reported as a sign of uterine torsion on magnetic resonance imaging (MRI) [7]. Although MRI is known as a better imaging modality for female pelvic anatomy, it may not be readily available in all parts of the world. Pelvic ultrasound is more readily available, however, it is less specific. The CT of our patient revealed an enlarged uterus measuring $26 \times 23.4 \times 16.5 \mathrm{~cm}$, heterogenous with amorphous calcification, $2.5 \mathrm{~cm}$ enhancing focus at the lower part of uterus, with the lower uterine segment torted along its long axis, incorporating bilateral adnexa. Bilateral ovarian veins also torted about the twisted pedicle. Enhancing tubular structures were noted in the lower aspects of uterus.

Due to the non-specific symptoms and signs, pre-operative clinical diagnosis is often difficult. However, this condition demands an early diagnosis and immediate surgical exploration to reduce the risks associated with ischemia and gangrene, also to preserve fertility in those of at reproductive age. More awareness and education are needed to recognize and identify the signs on imaging to assist in early diagnosis and definitive treatment.

Counselling of surgical options takes into consideration the age of the patient, desire for fertility, associated pathology and viability of adnexa and uterus. Total hysterectomy and bilateral salpingo-oophorectomy are usually performed in those who have completed family, elderly, and whose uterus and/or adnexa had undergone gangrene and necrosis due to prolonged torsion. In women of reproductive age group and who require fertility, conservative surgery is aimed for as much as possible, weighing the risks and benefit of fertility versus complications from an ischemic tissue. This usually included myomectomy or cystectomy. Our patient underwent total hysterectomy and bilateral salpingo-oophorectomy as the uterus and bilateral adnexa were ischemic and gangrenous looking.

\section{Acknowledgments}

Not applicable.

\section{Financial Disclosure}

Non to declare.

\section{Conflict of Interest}

None.

\section{Informed Consent}

Not applicable.

\section{Author Contributions}

Dr Ping Ling Ho: data and case collection, design and writeup, and finalization of write-up. Dr SHM Siraj, and Prof Dr Bernard Chern: supervision and final editing.

\section{References}

1. Davies JH. Case report: Torsion of a nongravid nonmyomatous uterus. Clin Radiol. 1998;53(10):780-782.

2. Varras M, Polyzos D, Alexopoulos C, Pappa P, Akrivis C. Torsion of a non-gravid leiomyomatous uterus in a patient with myotonic dystrophy complaining of acute urinary retention: anaesthetic management for total abdominal hysterectomy. Clin Exp Obstet Gynecol. 2003;30(2-3):147150 .

3. Holbrook J, Schneller C, Lapping-Carr G, Hageman JR, Lo A. A 3-year-old female with abdominal pain, vomiting, and an abdominal mass. Pediatr Ann. 2015;44(1):e69.

4. Grover S, Sharma Y, Mittal S. Uterine torsion: a missed diagnosis in young girls? J Pediatr Adolesc Gynecol. 2009;22(1):e5-8.

5. Yitta S, Hecht EM, Slywotzky CM, Bennett GL. Added value of multiplanar reformation in the multidetector CT evaluation of the female pelvis: a pictorial review. Radiographics. 2009;29(7):1987-2003.

6. Nicholson WK, Coulson CC, McCoy MC, Semelka RC. Pelvic magnetic resonance imaging in the evaluation of uterine torsion. Obstet Gynecol. 1995;85(5 Pt 2):888890.

7. Luk SY, Leung JL, Cheung ML, So S, Fung SH, Cheng $\mathrm{SC}$. Torsion of a nongravid myomatous uterus: radiological features and literature review. Hong Kong Med J. 2010;16(4):304-306. 